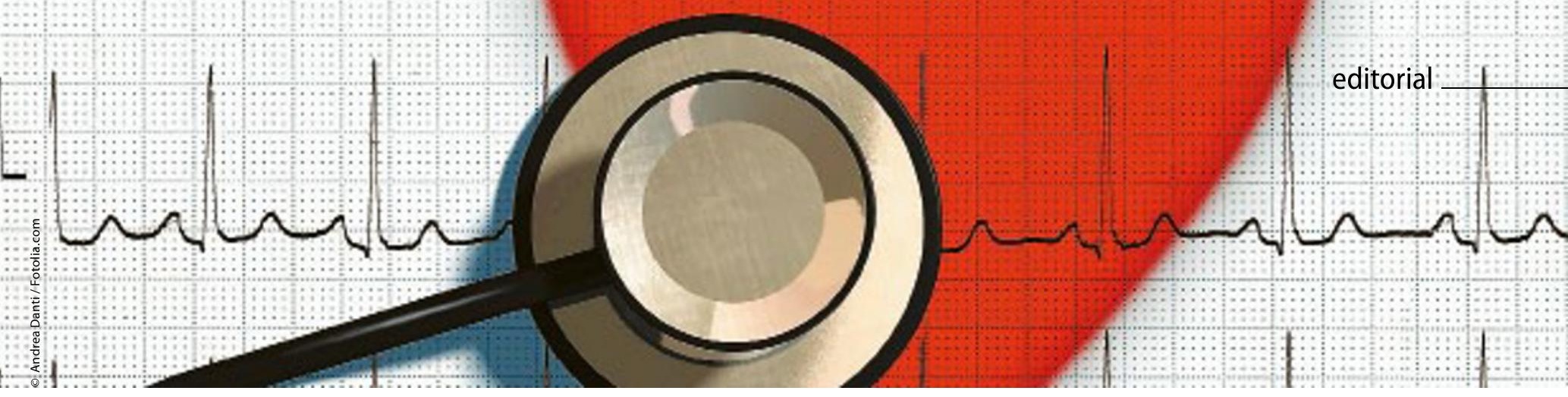

\title{
Nur schlechte Nachrichten sind gute Nachrichten
}

Sie erinnern sich wahrscheinlich noch an die Schlagzeile der Wochenzeitung DIE ZEIT vor einem Jahr: „Hunderte von tödlichen Blutungen durch Dabigatran bei der Behandlung von Vorhofflimmern." Diese Meldung lief über alle Presseagenturen und fand sich in fast allen Zeitungen und Zeitschriften. Zum damaligen Zeitpunkt waren in Deutschland etwa 70.000 Patienten mit Dabigatran behandelt und der Firma Boehringer-Ingelheim sowie den Behörden bundesweit vier tödliche Blutungen unter der Einnahme von Dabigatran kommuniziert worden.

Seither hat Boehringer-Ingelheim weltweit alle Fälle von schwerwiegenden und tödlichen Blutungen unter der Behandlung mit Dabigatran gesammelt und veröffentlicht diese für Ärzte auf einer monatlichen Basis bezogen auf 100.000 behandelte Patienten. Dabei zeigt sich, dass die Raten an schwerwiegenden und tödlichen Blutungen deutlich niedriger sind als in der RE-LY-Studie und dass die Zahlen insge samt abnehmend sind. Dies hat wahrscheinlich damit zu tun, dass die verschreibenden Ärzte gelernt haben, die Kontraindikationen zu beachten. Besonders beruhigend ist eine Mitteilung der US-amerikanischen Food- and Drug-Administration (FDA), dem Äquivalent des deutschen Bundesinstituts für Arzneimittel und Medizinalprodukte (BfArM): Die FDA hat Zugang zu den Datenbanken von großen Versicherungen inklusive Medicaid und Medicare in den Vereinigten Staaten und hat die Daten von mehreren hunderttausend Patienten auswerten können, denen erstmalig Dabigatran in der hohen Dosis von 2x $150 \mathrm{mg}$ oder Warfarin bei Vorhofflimmern zur Schlaganfallprophylaxe verordnet worden war. Diese Beobachtungsstudie zeigt, dass die Häufigkeit schwerwiegender Blutungskomplikationen unter Warfarin 1,8- bis 2,6-mal häufiger war als unter Dabigatran. Unterteilt man die Blutungskomplikationen war das Risiko von gastrointestinalen Blutungen für Warfarin um den Faktor 1,6 bis 2,2 höher als unter Dabigatran und das Risiko intrakranieller Blutungen 2,1- bis 3-fach höher.

Interessanterweise fand diese sehr positive Nachricht keinen Eingang in die Presselandschaft. Lediglich Fachpressedienste kommunizierten diese wichtige Beobachtung. Fast zeitgleich mit den positiven Meldungen der US-amerikanischen Zulassungsbehörde tat sich dann wieder etwas im deutschen Blätter-

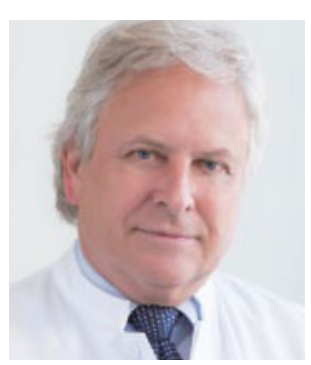

Prof. Dr. med. Hans-Christoph Diener, Essen

Direktor der Klinik für Neurologie,

Universitätsklinikum Essen

E-Mail:h.diener@uni-essen.de

wald: Und zwar wurde mitgeteilt, dass in den Vereinigten Staaten bereits 150 Prozesse wegen Blutungskomplikationen unter Dabigatran anhängig seien. Um dies richtig einschätzen zu können, muss man mit dem US-amerikanischen Rechtssystem vertraut sein. Denn es erlaubt Rechtsanwaltskanzleien Werbespots im Fernsehen zu schalten und Zeitungsanzeigen aufzugeben, in denen sie Patienten auffordern, bei Nebenwirkungen einer medikamentösen Therapie einen Rechtsanwalt aufzusuchen, um zu prüfen, ob sie den Hersteller des entsprechenden Medikamentes verklagen können. Begründet werden einige der Klagen damit, dass es für Dabigatran kein Antidot gäbe. Umfangreiche Analysen aus der RE-LY-Studie und anderen Studien mit Dabigatran zeigen aber, dass die Prognose bei Patienten, die eine schwerwiegende Blutung unter Dabigatran erlitten, deutlich besser ist als bei den Patienten, die eine entsprechende Blutung unter Warfarin hatten, obwohl es hier vermeintlich mit Vitamin $\mathrm{K}$ ein Antidot gibt. Interessant ist auch hier wieder die $\mathrm{Be}$ obachtung, dass nur die negative Nachricht Eingang in die $\mathrm{Pu}-$ blikumspresse findet, während die positiven Nachrichten von Zulassungsbehörden untergehen.

Hans-Christoph Diener 